TITLE:

\title{
A pn-junction between chalcopyrite phosphide semiconductors for photovoltaic application
}

$\operatorname{AUTHOR}(S)$ :

Nakatsuka, Shigeru; Kazumi, Kenji; Nose, Yoshitaro

\section{CITATION:}

Nakatsuka, Shigeru ...[et al]. A pn-junction between chalcopyrite phosphide

semiconductors for photovoltaic application. Japanese Journal of Applied Physics 2019, 58(7): 075508.

\section{ISSUE DATE:}

2019-07-01

URL:

http://hdl.handle.net/2433/243853

\section{RIGHT:}

This is a peer-reviewed, un-copyedited version of an article accepted for publication/published in Japanese Journal of Applied Physics. IOP Publishing Ltd is not responsible for any errors or omissions in this version of the manuscript or any version derived from it. The Version of Record is available online at https://doi.org/10.7567/1347-4065/ab28af.; The full-text file will be made open to the public on 3 July 2020 in accordance with publisher's 'Terms and Conditions for Self-Archiving'.; この論文は出版社版でありません。引用の際には出版社版をご確認ご利用ください。; This is not the published version. Please cite only the published version. 


\title{
A pn-junction between chalcopyrite phosphide semiconductors for photovoltaic application
}

\author{
Shigeru Nakatsuka, Kenji Kazumi and Yoshitaro Nose \\ Department of Materials Science and Engineering, Kyoto University, Kyoto, Japan
}

\section{Abstract}

We report on the fabrication of pn-junction between II-IV-V $\mathrm{V}_{2}$ type compounds with chalcopyrite crystal structure such as $\mathrm{CdSnP}_{2}$ and $\mathrm{ZnSnP}_{2}$ for photovoltaic application. In the fabrication process, $\mathrm{Cd}-\mathrm{Sn}$ precursor thin films were prepared on $\mathrm{ZnSnP}_{2}$ bulk crystals grown by flux method and the precursor thin films reacted with phosphorus gas to form $\mathrm{CdSnP}_{2} / \mathrm{ZnSnP}_{2}$ junction. STEM-EDX analysis and SAED patterns revealed that $\mathrm{CdSnP}_{2}$ was epitaxially grown on $\mathrm{ZnSnP}_{2}$ bulk crystals, indicating that the favourable junction was obtained in the view point of carrier transport. In addition, $\mathrm{Zn}$ was also detected in the region of $\mathrm{CdSnP}_{2}$ thin film due to the diffusion of $\mathrm{Zn}$ during phosphidation. This suggests the formation of solid solution $(\mathrm{Cd}, \mathrm{Zn}) \mathrm{SnP}_{2}$ between $\mathrm{ZnSnP}_{2}$ and $\mathrm{CdSnP}_{2}$, leading to realization of homojunction. In the $J-V$ measurements of the $n-(\mathrm{Cd}, \mathrm{Zn}) \mathrm{SnP}_{2} / p$ $\mathrm{ZnSnP}_{2}$ junction, a rectifying behavior was observed. The results in this work are cornerstones for photovoltaic application using II-IV $-\mathrm{V}_{2}$ type compound semiconductors including phosphides. 


\section{Introduction}

Thin film solar cells based on compound semiconductors are one of the promising candidates for the alternative to Si-based solar cells. For example, $\mathrm{CuIn}_{1-x} \mathrm{Ga}_{x} \mathrm{Se}_{2}$ (CIGS) and CdTe recorded high efficiencies of $22.9 \%^{1}$ and $22.1 \%^{2}$, respectively, which are comparable to that of multicrystalline-Si solar cells, $22.3 \%^{3}$. However, the usage of rare or toxic elements, such as $\mathrm{In}, \mathrm{Ga}, \mathrm{Cd}$ and $\mathrm{Te}$, prevents the widespread use of these compound semiconductors. Although chalcogenide compounds including S, Se and Te have been mainly researched in the field of thin film solar cells, II-IV- $V_{2}$ type pnictide compounds, such as $\mathrm{ZnSnP}_{2}{ }^{4-6}$ and $\mathrm{ZnSnN}_{2}{ }^{7-9}$ attract much attention as solar absorbing materials in recent years.

Our research group focused on $\mathrm{ZnSnP}_{2}$ with the chalcopyrite structure, which has a suitable direct bandgap of $1.6 \mathrm{eV}^{10-16}$ for photovoltaic devices. Based on the ShockleyQueisser limit, ${ }^{17}$ the theoretical conversion efficiency of about $30 \%$ is calculated in the single-junction solar cell using $\mathrm{ZnSnP}_{2}$ under the condition of $\mathrm{AM} 1.5 \mathrm{G}$ solar spectrum. ${ }^{18}$ In addition, the absorption coefficient of $\mathrm{ZnSnP}_{2}$ was reported to be approximately $10^{5}$ $\mathrm{cm}^{-1}$ in the visible light range, ${ }^{18,19}$ which enables thin film device. For electronic properties, non-doped $\mathrm{ZnSnP}_{2}$ shows a p-type conduction with the carrier concentration of $10^{16}-10^{18} \mathrm{~cm}^{-3} \cdot{ }^{10-16}$ The dominant intrinsic defects are antisite atoms reported based 
on the first principle calculation by Kumagai et al. ${ }^{20}$ Our research group also reported that $\mathrm{ZnSnP}_{2}$ solar cells recorded $3.44 \%$ with the structure of $\mathrm{Al} / \mathrm{Al}$-doped $\mathrm{ZnO} / \mathrm{ZnO} /(\mathrm{Cd}, \mathrm{Zn}) \mathrm{S} / \mathrm{ZnSnP} / \mathrm{Cu}$. The cell parameters such as short circuit current density, $J_{\mathrm{SC}}$, open circuit voltage, $V_{\mathrm{OC}}$, and fill factor, $F F$, were $12.3 \mathrm{~mA} / \mathrm{cm}^{2}, 0.472 \mathrm{~V}$ and 0.594 , respectively. ${ }^{4}$ Considering the bandgap of $\mathrm{ZnSnP}_{2}, V_{\mathrm{OC}}$ was largely lower than expected. The measurements by X-ray photoelectron spectroscopy (XPS) revealed a large conduction band offset, $-1.2 \mathrm{eV}$ at the heterojunction between $\mathrm{CdS}$ and $\mathrm{ZnSnP}_{2} .{ }^{21}$ It is considered that $(\mathrm{Cd}, \mathrm{Zn}) \mathrm{S}$ used in the best cell also has a large conduction band offset with $\mathrm{ZnSnP}_{2}$, which limits $V_{\text {OC. }}$ Therefore, the improvement of the band offset in $\mathrm{ZnSnP}_{2}$ solar cells is required.

In this study, $\mathrm{CdSnP}_{2}$ was focused on as a $n$-type material alternative to $(\mathrm{Cd}, \mathrm{Zn}) \mathrm{S}$. $\mathrm{CdSnP}_{2}$ also has a chalcopyrite structure and a bandgap of $1.17 \mathrm{eV} .^{22}$ Hinuma et al. carried out the first-principles calculation in $\mathrm{ZnSnP}_{2}, \mathrm{CdSnP}_{2}$ and related materials to obtain their band structures. ${ }^{23}$ They revealed that the conduction band offset between $\mathrm{CdSnP}_{2}$ and $\mathrm{ZnSnP}_{2}$ was expected to be $-0.5 \mathrm{eV}$. We thus consider an application of $\mathrm{CdSnP}_{2} / \mathrm{ZnSnP}_{2}$ pn-heterojunction to photovoltaics and investigate the diode characteristics and the interface structure with nanoscale. 


\section{Experimental method}

$\mathrm{CdSnP}_{2} / \mathrm{ZnSnP} 2$ junction was prepared by the formation of $\mathrm{CdSnP}_{2}$ thin film on the surface of $\mathrm{ZnSnP}_{2}$ bulk crystal fabricated by flux method. The details of experimental condition for bulk crystal growth were well described in our previous work. ${ }^{12}$ The raw materials such as zinc shots (99.99\%, Kojundo Chemical Laboratory), tin shots (99.99\%, Kojundo Chemical Laboratory) and red phosphorus flakes (99.9999\%, Kojundo Chemical Laboratory) were sealed in an evacuated quartz ampule under the pressure of $10^{-2} \mathrm{~Pa}$. The nominal composition was determined to be $92 \mathrm{~mol} \% \mathrm{Sn}$ in $\mathrm{Sn}-\mathrm{ZnP}_{2}$ pseudobinary system. Then, sample was unidirectionally solidified from the bottom with the average cooling rate of about $0.7^{\circ} \mathrm{C} / \mathrm{h}$ after heated up to $700{ }^{\circ} \mathrm{C}$ for homogenization. The grown $\mathrm{ZnSnP}_{2}$ crystals were cut into several wafers in perpendicular to the growth direction. The surface of each wafer was mechanically polished to obtain mirror surface. And the $\mathrm{ZnSnP}_{2}$ wafers were etched by immersing in $1 / 2$ diluted aqua regia $\left(\mathrm{HCl}: \mathrm{HNO}_{3}\right.$ $=3: 1)$ for $15 \mathrm{~min}$ to remove the damaged layer derived from mechanical polishing. ${ }^{5}$

In our previous work, the fabrication process for $\mathrm{CdSnP}_{2}$ thin films was already established using phosphidation method, where Cd-Sn precursor thin films react with phosphorus gas. ${ }^{24} \mathrm{Cd}-\mathrm{Sn}$ thin films with the thickness of $100 \mathrm{~nm}$ were prepared as a 
precursor on the polished and etched surfaces of $\mathrm{ZnSnP}_{2}$ bulk crystals by co-deposition with DC magnetron sputtering using Cd (99.9\%, Kojundo Chemical Laboratory) and Sn (99.99\%, Furuuchi Chemical Corporation) targets. The sputtering period was 15 min and the pressure of Ar gas was $0.8 \mathrm{~Pa}$. Considering the higher vapor pressure of $\mathrm{Cd}$, the molar ratio $[\mathrm{Cd}] /[\mathrm{Sn}]$ was controlled to be about 1.2 in the precursor thin films.

Subsequently, Cd-Sn thin films reacted with phosphorus gas in order to obtain $\mathrm{CdSnP}_{2} / \mathrm{ZnSnP}_{2}$ heterojunction. The substrate temperature was about $350{ }^{\circ} \mathrm{C}$ and the duration time for phosphidation was $30 \mathrm{~min}$. In the experiments, we used two-phase sample with $\mathrm{Sn}$ and $\mathrm{Sn}_{4} \mathrm{P}_{3}$ as a phosphorus source and phosphorus gas was transported to the precursor samples using deoxygenated Ar gas. The partial pressure of phosphorus was controlled to be approximately $10^{-2}$ atm by controlling the temperature of the two-phase sample. The cross-sectional observation was carried out using scanning transmission electron microscopy with energy dispersive X-ray analysis (STEM-EDX, JEM-2100F, JEOL) and selected area electron diffraction (SAED) patterns were obtained using a transmission electron microscope (TEM, JEM-2100F, JEOL) to investigate the interfacial structure. The thin samples for the cross-sectional observation were prepared by focused ion beam process with Ga ion source (FIB, SMI 9200 SII). In order to investigate the current density-voltage $(J-V)$ characteristics of $\mathrm{CdSnP}_{2} / \mathrm{ZnSnP}_{2}$ junction, $\mathrm{Sn}$ and $\mathrm{Cu}$ 
electrodes with the thickness of about $0.5 \mu \mathrm{m}$ were deposited by DC-sputtering on $\mathrm{CdSnP}_{2}$ and $\mathrm{ZnSnP}_{2}$ sides, respectively, for Ohmic contacts. Sn (99.99\%, Furuuchi Chemical Corporation) and $\mathrm{Cu}(99.99 \%$, Furuuchi Chemical Corporation) were used as the target materials. And the sample was sealed in an evacuated quartz ampule and annealed at $300{ }^{\circ} \mathrm{C}$ for $20 \mathrm{~min} .{ }^{5}$ The $J-V$ characteristics of $\mathrm{CdSnP}_{2} / \mathrm{ZnSnP}_{2}$ junction were measured in the voltage range from $-1.5 \mathrm{~V}$ to $1.5 \mathrm{~V}$ using a digital sourcemeter (Keithley, model2400).

\section{Results and discussion}

Figure 1 shows cross-sectional STEM images and corresponding elemental mappings of the samples before and after phosphidation. Before phosphidation, it is observed that the Cd-Sn precursor thin film was deposited on the $\mathrm{ZnSnP}_{2}$ bulk crystal with rough morphology as shown in Figure 1 (a). From the contrast between Cd-L and Sn-L in the elemental mappings, it can be recognized that $\mathrm{Cd}$ and $\mathrm{Sn}$ particles were separated, particularly understandable around center area, which was also observed on the glass substrates in our previous work ${ }^{24}$. It is consistent with that the phase diagram of the CdSn system is a typical eutectic diagram. ${ }^{25}$ Figure 1 (b) shows the formation of compounds or alloys containing $\mathrm{Cd}, \mathrm{Sn}$ and $\mathrm{P}$ on the $\mathrm{ZnSnP}_{2}$ bulk crystal by phosphidation. This 
indicates the formation of $\mathrm{CdSnP}_{2} / \mathrm{ZnSnP} 2$ junction when considering together with SAED pattern shown later. Comparing the STEM images before and after phosphidation, it is observed that the original boundary between $\mathrm{Cd}-\mathrm{Sn}$ precursor thin film and $\mathrm{ZnSnP} 2$ bulk crystal changed from flat to curved boundary. In addition, $\mathrm{Zn}$ was also detected in the Cd-Sn-P region. On the other hand, the junction was inhomogeneously formed and the precipitation of Sn was observed at the interface as shown in Figure 1 (c). Figures 1 (b) and 1 (c) are different areas in the same sample. The realization of homogeneous interfacial structure is thus required as future works. The phosphidation is unsuitable for smooth interface because of the roughness of Cd-Sn precursor films, and then deposition methods such as molecular beam epitaxy (MBE) is desirable.

Then, TEM observation was carried out and SAED patterns were taken from each region around the interface to investigate the crystal structure, as shown in Figure 2. $\mathrm{ZnSnP}_{2}<110>$ was selected as the zone axis. The SAED patterns in $\mathrm{ZnSnP}_{2}$ and $\mathrm{Cd}-\mathrm{Sn}-$ $\mathrm{P}$ regions show the same pattern, indicating that the crystal structure in the Cd-Sn-P region is same as $\mathrm{ZnSnP}_{2}$. In other words, $\mathrm{CdSnP}_{2}$ with chalcopyrite structure was grown on $\mathrm{ZnSnP}_{2}$ by phosphidation. In addition, both phosphides show the same orientation and thus the epitaxial relationship between $\mathrm{CdSnP}_{2}$ and $\mathrm{ZnSnP}_{2}$ is understood. From the viewpoint of carrier transport in photovoltaic devices, such an epitaxial growth is 
considered to be favourable to achieve a higher conversion efficiency. On the other hand, the SAED pattern at the interface shown in Figure 2 (c) seems to be an overlap of those taken from $\mathrm{ZnSnP}_{2}$ and $\mathrm{CdSnP}_{2}$ regions. To investigate the detailed structure at the $\mathrm{CdSnP}_{2} / \mathrm{ZnSnP}_{2}$ interface, the composition analysis based on STEM-EDX was carried out. Figure 3 shows the composition distribution across the interface of $\mathrm{CdSnP}_{2} / \mathrm{ZnSnP}_{2}$. The composition was evaluated based on the Cliff-Lorimer method, where Sn was selected as the base element and $k$-factor of $\mathrm{Zn}$ and $\mathrm{P}$ were calculated using the $\mathrm{ZnSnP} 2$ bulk crystal region, and that of $\mathrm{Cd}$ was obtained using the $\mathrm{CdSnP}_{2}$ bulk crystal sample separately prepared. The compositions of $\mathrm{P}$ and $\mathrm{Sn}$ are approximately 50 and 25 at. \%, respectively, independent of the scanned positions except for the surface, and the composition change of $\mathrm{Zn}$ and $\mathrm{Cd}$ is observed. This means that the formation of the solid solutions between $\mathrm{CdSnP}_{2}$ and $\mathrm{ZnSnP}_{2},(\mathrm{Cd}, \mathrm{Zn}) \mathrm{SnP}_{2}$, which is also supported by the SAED pattern at the interface as shown in Figure 2 (c). The concentration change is continuous and the interface with less lattice defects might be formed. In addition, in the $\mathrm{CdSnP}_{2}$ region, the concentration of $\mathrm{Zn}$ is at least 5 at.\%, which correspond to the mole fraction $[\mathrm{Zn}] /([\mathrm{Cd}]+[\mathrm{Zn}])$ of 0.2 . On the other hand, $\mathrm{Cd}$ was hardly detected in the $\mathrm{ZnSnP} 2$ bulk crystal side. In this work, the heat treatment was not carried out after the formation of $\mathrm{CdSnP}_{2}$ and the above asymmetric composition distribution is due to the $\mathrm{Zn}$ diffusion 
into $\mathrm{CdSnP}_{2}$ region during phosphidation. From the above experimental results, the obtained $(\mathrm{Cd}, \mathrm{Zn}) \mathrm{SnP}_{2} / \mathrm{ZnSnP} \mathrm{P}_{2}$ interface can be recognized as a homointerface due to both compounds thermodynamically-indistinguishable although it is considered a heterointerface at first sight. It is thus expected that the above diffusion behavior is controlled by experimental conditions such as heat treatment during phosphidation and preparation of precursor thin films, and it is a key to obtain favorable interface for photovoltaic application.

Finally, the $J-V$ characteristics were measured using the sample with the structure of $\mathrm{Sn} / \mathrm{CdSnP}_{2} / \mathrm{ZnSnP} / \mathrm{Cu}$. Figure 4 shows the $J-V$ curves in linear and log scale. As shown in these figures, a rectifying behavior is observed, suggesting the formation of a pnjunction at the $n-(\mathrm{Cd}, \mathrm{Zn}) \mathrm{SnP}_{2} / p-\mathrm{ZnSnP}_{2}$ interface because $\mathrm{Cu} / \mathrm{ZnSnP} \mathrm{P}_{2}$ and $\mathrm{Sn} / \mathrm{CdSnP}_{2}$ junctions have been known to show an Ohmic behavior in our preliminary works. The conventional $J-V$ relationship for single-diode expressed by the equation (1) was fitted to the measured curve,

$$
J=J_{0}\left\{\exp \left[\frac{q\left(V-R_{\mathrm{s}} J\right)}{N k T}-1\right]\right\}+\frac{V-R_{\mathrm{s}} J}{R_{\mathrm{sh}}}
$$

where $k$ is the Boltzmann constant and $T$ is the temperature. Consequently, the diode parameters such as saturation current density, $J_{0}$, ideality factor, $N$, series resistance, $R_{\mathrm{S}}$, 
and shunt resistance, $R_{\mathrm{Sh}}$, were evaluated to be $1.41 \times 10^{-8} \mathrm{~A} \mathrm{~cm}^{-2}, 1.66,68.9 \Omega \mathrm{cm}^{2}$ and $8.50 \times 10^{5} \Omega \mathrm{cm}^{2}$, respectively. Comparing the parameters for CdS/CIGS junction ${ }^{26}$, the series resistance and the saturation current density are relatively high for photovoltaic application. One of the reasons is inhomogeneity of $(\mathrm{Cd}, \mathrm{Zn}) \mathrm{SnP}_{2} / \mathrm{ZnSnP}_{2}$ junction as described in the STEM analysis and it should be established to prepare the uniform junction over the entire interface. The high resistivity of $\mathrm{CdSnP} 2$ prepared by phosphidation $^{24}$, resulting in high series resistance in Figure 4, should be also considered. The above improvement is required for the detailed analysis for interface such as capacitance-voltage measurements and the device fabrication as future works. 


\section{Conclusions}

In this study, Cd-Sn precursor thin films were prepared on $\mathrm{ZnSnP}_{2}$ bulk crystals grown by flux method and then $\mathrm{CdSnP}_{2}$ thin films were formed by the phosphidation method for fabrication of $\mathrm{CdSnP}_{2} / \mathrm{ZnSnP}_{2}$ junction. STEM-EDX analysis and SAED patterns revealed that $\mathrm{CdSnP}_{2}$ with chalcopyrite structure was epitaxially grown on $\mathrm{ZnSnP} 2$ bulk crystals. In addition, the diffusion of $\mathrm{Zn}$ into $\mathrm{CdSnP}_{2}$ region during phosphidation was observed, while the concentrations of $\mathrm{P}$ and $\mathrm{Sn}$ are independent of positions around the interface. The above results indicate that favourable junction was obtained due to the formation of solid solution, $(\mathrm{Cd}, \mathrm{Zn}) \mathrm{SnP}_{2}$, in the view point of carrier transport. However, $n$-(Cd, $\mathrm{Zn}) \mathrm{SnP}_{2} / p-\mathrm{ZnSnP}_{2}$ junction was inhomogeneous and $\mathrm{Sn}$ was precipitated in some areas around the interface, which should be improved in future works. In the $J-V$ characteristic of the junction, a rectifying behavior was observed although the series resistance and the saturation current density were relatively high. It is thus concluded that the $n-(\mathrm{Cd}, \mathrm{Zn}) \mathrm{SnP}_{2} / p-\mathrm{ZnSnP} 2$ junction obtained in this work can be recognized as a pnhomojunction and this work is one of fundamentals to have more choices for solar absorbing materials including II-IV-V $\mathrm{V}_{2}$ type compounds. 


\section{Acknowledgements}

The authors would like to express their gratitude to Dr. R. Katsube (Kyoto Univ.) for his fruitful discussions. This work was financially supported by JST CREST Grant Number JPMJCR17J2, The Mitsubishi Foundation and Grant-in-Aid for JSPS Research

Fellow Number 16J09443. This work was also supported by the Collaborative Research

Project of Laboratory for Materials and Structures, Institute of Innovative Research, Tokyo Institute of Technology. 


\section{References}

${ }^{1}$ Solar Frontier. Solar Frontier Achieves World Record Thin-Film Solar Cell Efficiency of 22.9\%. http://www.solar-frontier.com/eng/news/2017/1220_press.html (accessed in August 2018).

${ }^{2}$ First Solar. First Solar Hits Record 22.1\% Conversion Efficiency for CdTe Solar Cell. https://www.greentechmedia.com/articles/read/First-Solar-Hits-Record-22.1-

Conversion-Efficiency-For-CdTe-Solar-Cell (accessed in August 2018).

${ }^{3}$ J. Benick, R. Müller, F. Schindler, A. Richter, H. Hauser, F. Feldmann, P. Krenckel, S.

Riepe, M. C. Schubert, M. Hermle, S. W. Glunz, Approaching 22\% Efficiency with Multicrystalline N-Type Silicon Solar Cells, 33rd European PV Solar Energy Conference and Exhibition, Amsterdam, Nertherlands 25-29 Sep (2017).

${ }^{4}$ S. Akari, J. Chantana, S. Nakatsuka, Y. Nose, and T. Minemoto, Sol. Energy Mater. Sol. Cells 174, 412 (2018).

${ }^{5}$ S. Nakatsuka, S. Akari, J. Chantana, T. Minemoto, and Y. Nose, ACS Appl. Mater. Interfaces 9, 33827 (2017).

${ }^{6}$ N. Yuzawa, J. Chantana, S. Nakatsuka, Y. Nose, and T. Minemoto, Curr. Appl. Phys. 17, 557 (2017).

${ }^{7}$ L. Lahourcade, N.C. Coronel, K.T. Delaney, S.K. Shukla, N.A. Spaldin, and H.A. 
Atwater, Adv. Mater. 25, 2562 (2013).

${ }^{8}$ P.C. Quayle, K. He, J. Shan, and K. Kash, MRS Commun. 3, 135 (2013).

${ }^{9}$ N. Feldberg, J.D. Aldous, W.M. Linhart, L.J. Phillips, K. Durose, P.A. Stampe, R.J.

Kennedy, D.O. Scanlon, G. Vardar, R.L. Field, T.Y. Jen, R.S. Goldman, T.D. Veal, and

S.M. Durbin, Appl. Phys. Lett. 103, (2013).

${ }^{10}$ A.A. Abdurakhimov, L. V. Kradinova, Z.A. Parimbekov, and Y. V. Rud', Sov. Phys.

Semicond. 16, 156 (1982).

${ }^{11}$ M.A. Ryan, M.W. Peterson, D.L. Williamson, J.S. Frey, G.E. Maciel, and B.A.

Parkinson, J. Mater. Res. 2, 528 (1987).

${ }^{12}$ S. Nakatsuka, H. Nakamoto, Y. Nose, T. Uda, and Y. Shirai, Phys. Status Solidi C 12, $520(2015)$

${ }^{13}$ D.O. Scanlon and A. Walsh, Appl. Phys. Lett. 100, 251911 (2012).

${ }^{14}$ P.K. Ajmera, H.Y. Shin, and B. Zamanian, Sol. Cells 21, 291 (1987).

${ }^{15}$ S. Nakatsuka, N. Yuzawa, J. Chantana, T. Minemoto, and Y. Nose, Phys. Status Solidi A 214, 1600650 (2017).

${ }^{16}$ K. Nakatani, T. Minemura, K. Miyauchi, K. Fukabori, H. Nakanishi, M. Sugiyama, and S. Shirakata, Jpn. J. Appl. Phys. 47, 5342 (2008).

${ }^{17}$ W. Shockley and H.J. Queisser, J. Appl. Phys. 32, 510 (1961). 
${ }^{18}$ T. Yokoyama, F. Oba, A. Seko, H. Hayashi, Y. Nose, and I. Tanaka, Appl. Phys.

Express 6, 61201 (2013).

${ }^{19}$ H.Y. Shin and P.K. Ajmera, Mater. Lett. 5, 211 (1987).

${ }^{20}$ Y. Kumagai, M. Choi, Y. Nose, and F. Oba, Phys. Rev. B 90, 125202 (2014).

${ }^{21}$ S. Nakatsuka, Y. Nose, and Y. Shirai, J. Appl. Phys. 119, 193107 (2016).

${ }^{22}$ J.L. Shay and J.H. Wernick, Chalcopyrite Semiconductors: Growth, Electronic

Properties, and Applications (Pergamon Press, Oxford, 1975).

${ }^{23}$ Y. Hinuma, F. Oba, Y. Nose, and I. Tanaka, J. Appl. Phys. 114, 043718 (2013).

${ }^{24}$ S. Nakatsuka, R. Inoue, and Y. Nose, ACS Appl. Energy Mater. 1, 1635 (2018).

${ }^{25}$ H. Okamoto, Phase Diagram for Binary Alloys, Desk Handbook (ASM International, 2000)

${ }^{26}$ P. Jackson, D. Hariskos, R. Wuerz, O. Kiowki, A. Bauer, T. M. Friedlmeier, and M.

Powalla, Phys. Status Solidi RRL, 9, 28 (2015). 
- Figure captions

Figure 1. Cross-sectional STEM images and corresponding elemental mappings of (a) before and (b), (c) after phosphidation.

Figure 2. SAED patterns taken from (a) $\mathrm{ZnSnP}_{2}$ region, (b) $\mathrm{Cd}-\mathrm{Sn}-\mathrm{P}\left(\mathrm{CdSnP}_{2}\right)$ region and (c) $\mathrm{CdSnP}_{2} / \mathrm{ZnSnP} 2$ interface.

Figure 3. Composition analysis at $\mathrm{CdSnP}_{2} / \mathrm{ZnSnP}_{2}$ interface based on STEM-EDX.

Figure 4. $J-V$ characteristics of $\mathrm{CdSnP}_{2} / \mathrm{ZnSnP}_{2}$ junction in (a) linear and (b) log scale. 


\section{- Figures}

(a)
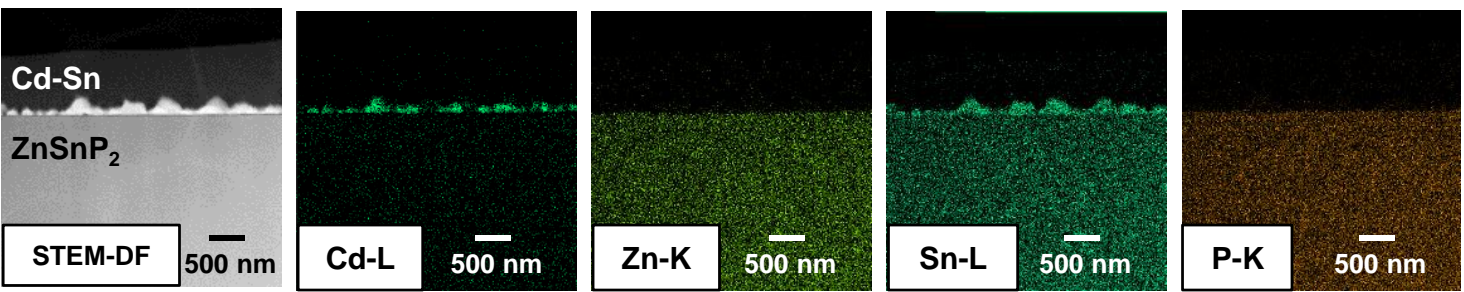

(b)
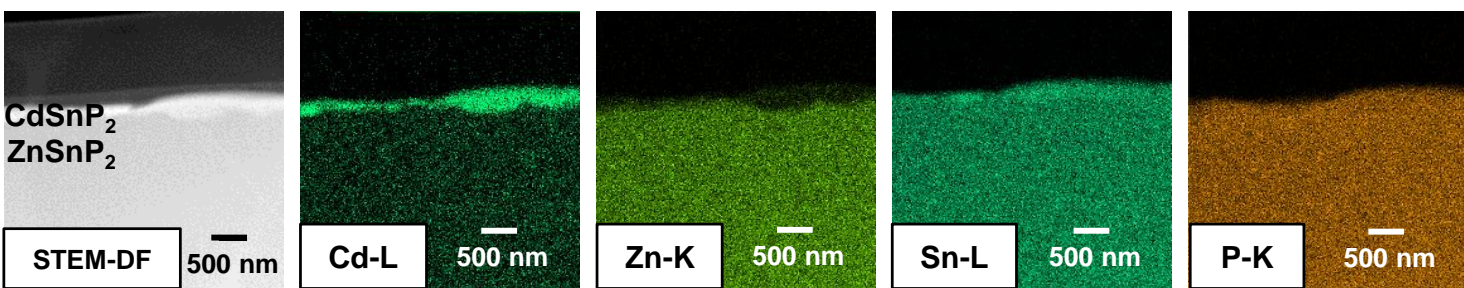

(c)
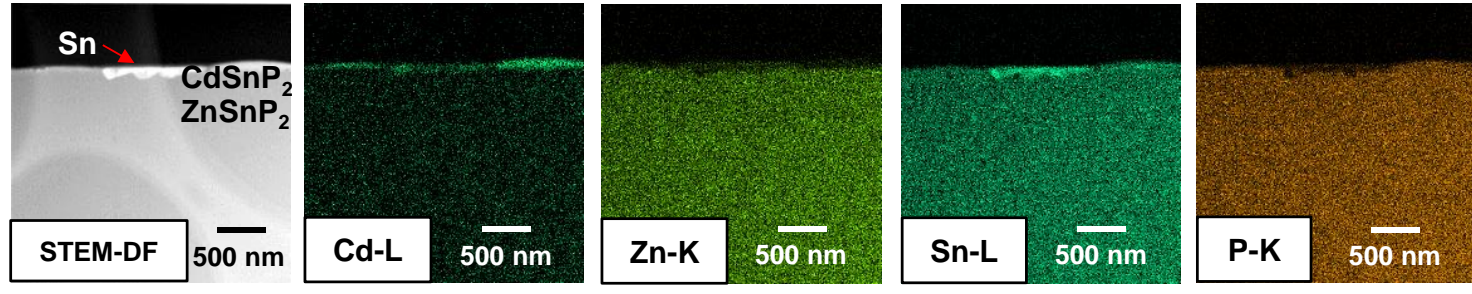

Fig. 1. Cross-sectional STEM images and corresponding elemental mappings of (a) before and (b), (c) after phosphidation. 

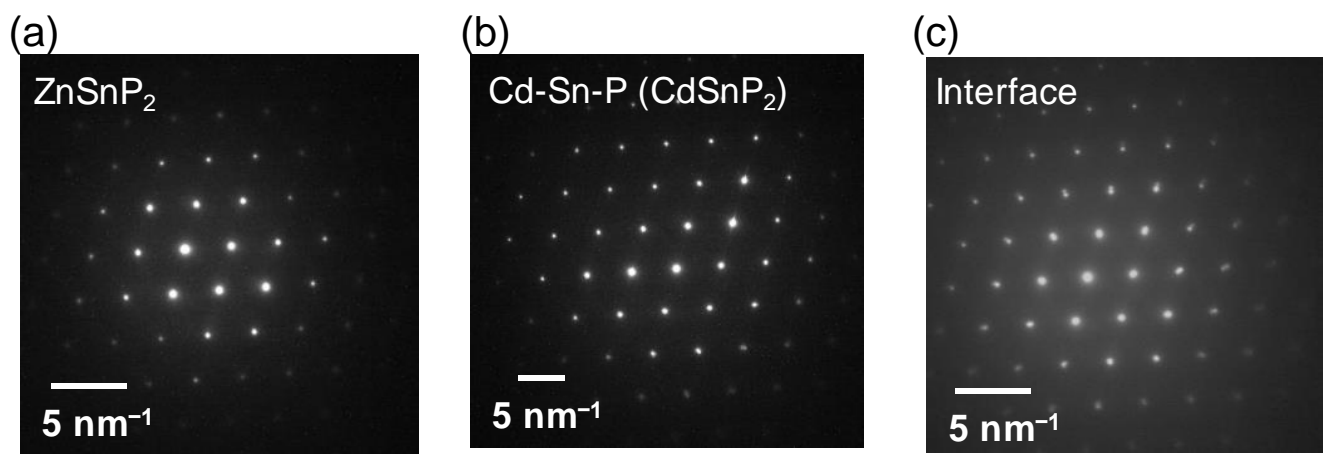

Fig. 2. SAED patterns taken from (a) $\mathrm{ZnSnP}_{2}$ region, (b) $\mathrm{Cd}-\mathrm{Sn}-\mathrm{P}\left(\mathrm{CdSnP}_{2}\right)$ region and (c) $\mathrm{CdSnP}_{2} / \mathrm{ZnSnP} \mathrm{P}_{2}$ interface. 


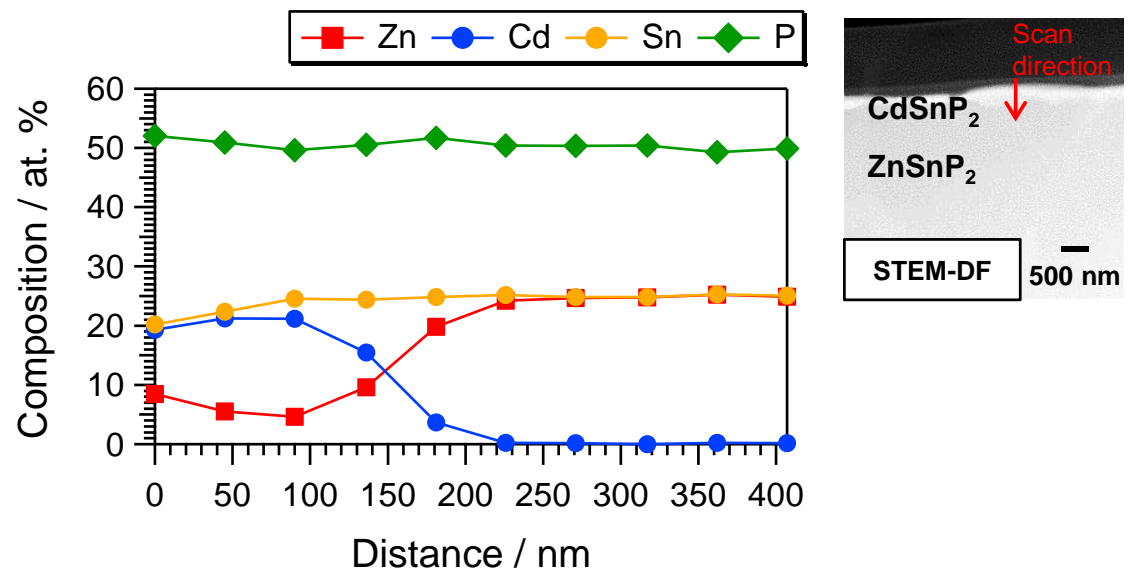

Fig. 3. Composition analysis at $\mathrm{CdSnP}_{2} / \mathrm{ZnSnP}_{2}$ interface based on STEM-EDX. 
(a)

(b)
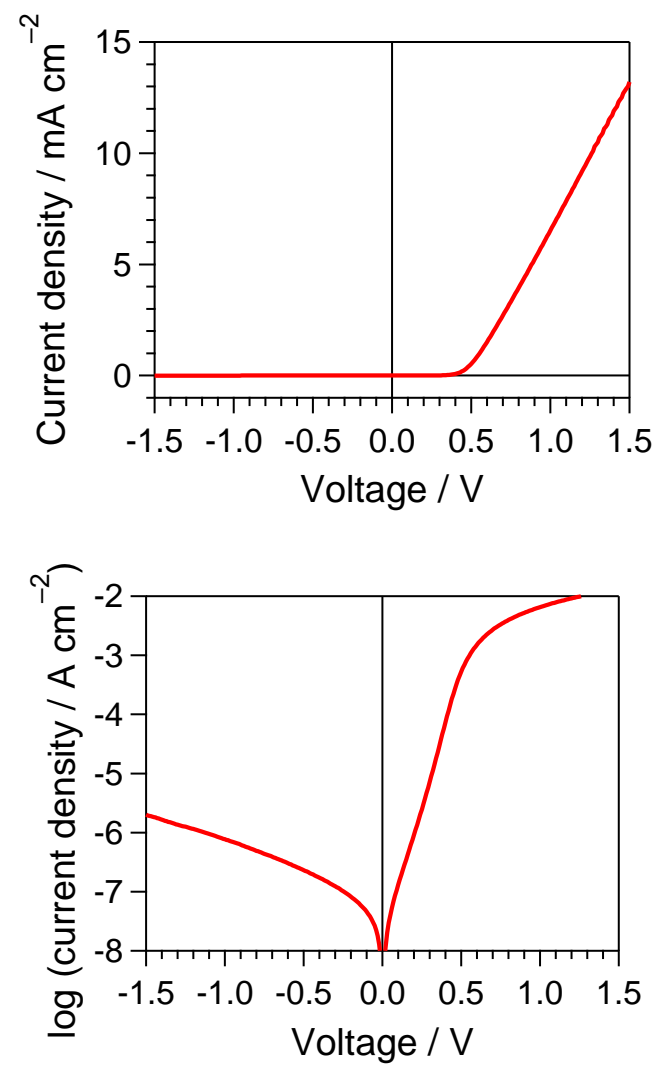

Fig. 4. $J-V$ characteristics of $\mathrm{CdSnP}_{2} / \mathrm{ZnSnP}_{2}$ junction in (a) linear and (b) log scale. 